\title{
Dénombrement des germes totaux et détermination du taux de contamination en staphylocoques coagulase-positive du fromage iranien frais, non salé, mis en vente dans l'agglomération de Téhéran
}

\author{
par \\ R. GHAZVINIAN*, A. FARKHONDEH*, Ph. LACHAL** \\ et M. KOHNECHAHRI*
}

\section{INTRODUCTION}

En Iran, l'origine de nombreuses entériostaphylotoxicoses étant imputées aux produits laitiers, diverses recherches ont été entreprises récemment à ce sujet $[1,2,3]$. Poursuivant les investigations dans ce domaine, on s'est attaché présentement à la recherche des staphylocoques présumés pathogènes dans le fromage frais iranien, fromage blanc de type bulgare, caillé-présure, produit artisanalement ou semi-industriellement $[4,5]$. La qualité des laits utilisés pour sa fabrication et les conditions hygiéniques de sa production en fait, en effet, suspecter à priori, une contamination staphylococcique importante.

\section{MATERIEL ET METHODES}

La collecte de 212 échantillons de ce fromage a été effectuée dans 36 échoppes situées dans les différents quartiers de Téhéran, entre octobre 1972 et mai 1973.

$50 \mathrm{~g}$ de fromage sont prélevés, en prenant toutes les mesures possibles de l'aseptie, et placés dans des flacons stériles numérotés. $\mathrm{Au}$ laboratoire, on a réalisé de façon stérile le broyage de $20 \mathrm{~g}$ de fromage dans $80 \mathrm{ml}$ d'eau distillée stérile à l'aide d'un mixer Warnig Blender. A partir de la fine suspension obtenue on a réalisé des

* Département de l'Hygiène alimentaire, Université de Téhéran.

** Docteur-Vétérinaire, Coopérant technique, appelé du Service National. 
dilutions allant de $10^{-2}$ à $10^{-\tau}$. Le dénombrement des germes totaux a été réalisé par la technique «Plate Count " en gélose nutritive ordinaire selon la norme FIL [6]. Pour la recherche et le dénombrement des staphylocoques, on a utilisé un milieu Baird-Parker BBL $[7,8]$ modifié. En effet, afin d'obtenir une coloration noire plus intense et plus brillante des colonies de staphylocoques, la concentration du milieu en Tellurite de Potassium a été doublée. L'ensemencement du milieu coulé en boîte de Petri est effectué de deux manières différentes pour chaque échantillon : d'une part, étalement de $0,1 \mathrm{ml}$ des solutions diluées, d'autre part, ensemencement " de contrôle » à l'öse bouclée à partir de la « solution mère » du mixer. La lecture des boîtes n'est faite qu'après $24 \mathrm{~h}$ et $48 \mathrm{~h}$ d'incubation à $37^{\circ} \mathrm{C}$. Les colonies suspectes sont repiquées sur milieu liquide Heart infusion Broth (Dif-Co) en tubes à essai. Ces tubes ont ensuite été placés à l'étuve à $37^{\circ} \mathrm{C}$ pendant $24 \mathrm{~h}$. Le caractère coagulase-positive est ensuite mis en évidence en mélangeant $0,5 \mathrm{ml}$ de plasma coagulase Rabbit (BBL) et $0,05 \mathrm{ml}$ de culture dans des tubes étroits placés dans un bain-marie à $37^{\circ} \mathrm{C}$. Les résultats sont lus tous les $1 / 4 \mathrm{~h}$ pendant $4 \mathrm{~h}$ et un dernier examen des tubes est effectué après $24 \mathrm{~h}$ pour dépister les réactions tardives.

La lysotypie des souches coagulase-positive isolées a été réalisée par le centre du typage bactériophagique de la Faculté de l'Hygiène Publique de l'Université de Téhéran.

\section{RESULTATS ET DISCUSSIONS}

Le tableau 1 montre le résultat du dénombrement des germes totaux des 212 échantillons soumis à l'analyse bactériologique.

TABLEAU 1

\begin{tabular}{c|c}
\hline $\mathrm{N}=$ Nombre de germes totaux $/ \mathrm{g}$ & p. 100 des échantillons \\
\hline $\mathrm{N}<100 \times 10^{6}$ & 4,3 \\
$100 \times 10^{6}<\mathrm{N}<1000 \times 10^{8}$ & 45,7 \\
$\mathrm{~N}>1000 \times 10^{8}$ & 50,0 \\
\hline
\end{tabular}

On voit en premier lieu que la flore mésophile totale de ces fromages est très importante. Parmi les échantillons présentant une numération totale supérieure à $10^{\circ}, 6,5$ p. 100 ont plus de $30 \times 10^{\circ}$ 
germes au gramme. Par ailleurs, 79 échantillons parmi les 212 analysés, soit 37,26 p. 100 ont permis l'isolement de staphylocoques coagulasepositive. Ces 79 échantillons se répartissent en fonction de la contamination staphylococcique comme le montre le tableau 2.

TABLEAU 2

\begin{tabular}{l|c|c}
\hline $\begin{array}{c}\text { Nombre de } \\
\text { staphylocoques/g }\end{array}$ & $\begin{array}{c}\text { Nombre } \\
\text { d'échantillons }\end{array}$ & $\begin{array}{c}\text { p.100 des échantillons } \\
\text { positifs }\end{array}$ \\
\hline $\mathrm{N}<10 \times 10^{3}$ & 13 & 16,46 \\
$10 \times 10^{3}<\mathrm{N}<100 \times 10^{3}$ & 48 & 60,76 \\
$100 \times 10^{3}<\mathrm{N}<500 \times 10^{3}$ & 15 & 18,99 \\
$\mathrm{~N}>500 \times 10^{3}$ & 3 & 3,79 \\
\hline Total & 79 & \\
\hline
\end{tabular}

Le tableau 3 exprime la répartition de ces échantillons selon la fraîcheur du fromage.

TABLEAU 3

\begin{tabular}{|c|c|c|}
\hline \multirow{2}{*}{$\begin{array}{r}\text { p. } 100 \text { des échantillons } \\
\text { positifs }\end{array}$} & \multicolumn{2}{|c|}{ Nombre de staphylocoques par g } \\
\hline & $\mathrm{N}<100 \times 10^{3}$ & $\mathrm{~N}>100 \times 10^{3}$ \\
\hline$\leqslant 12 \mathrm{~h}$ & 54,8 & 47 \\
\hline 12 à $24 \mathrm{~h}$ & 3,3 & 11,8 \\
\hline$\geqslant 24 \mathrm{~h}$ & 41,9 & 41,2 \\
\hline
\end{tabular}

Il convient de noter à ce propos que tous les échantillons comptant plus de $200 \times 10^{3}$ staphylocoques coagulase-positive/g ont au moins 24 h d'âge. 
Le tableau 4 permet de comparer le résultat de numération de la flore totale et celui de la détermination du taux de contamination en staphylocoques coagulase-positive.

TABLEAU 4

\begin{tabular}{l|c|c}
\hline \multirow{2}{*}{ Numération totale } & \multicolumn{2}{|c}{ Contamination en staphylocoques coagulase-positive } \\
\cline { 2 - 3 } & $\mathrm{N}<100 \times 10^{3}$ & $\mathrm{~N}>100 \times 10^{3}$ \\
\hline $\mathrm{N}<100 \times 10^{6}$ & 0 & 20 \\
$100 \times 10^{6}<\mathrm{N}<10^{\circ}$ & 46,3 & 33,3 \\
$\mathrm{~N}>10^{\circ}$ & 53,7 & 46,7 \\
\hline
\end{tabular}

Le tableau 5 montre le pourcentage des types bactériologiques des staphylocoques isolés des fromages frais.

TABLEAU 5

\begin{tabular}{|c|c|c|}
\hline Groupe & Numéro des échantillons & Pourcentage \\
\hline \multicolumn{3}{|l|}{ Groupe I : } \\
\hline $\begin{array}{l}52,79,80 \\
29,52,52^{\AA}, 79,80 \\
80 \\
52^{\mathrm{A}}\end{array}$ & $\begin{array}{l}1 \\
1 \\
1 \\
1\end{array}$ & $\begin{array}{l}1.2 \\
1.2 \\
1.2 \\
1.2\end{array}$ \\
\hline Total groupe & 4 & $\overline{4.8}$ \\
\hline \multicolumn{3}{|l|}{ Groupe II : } \\
\hline $\begin{array}{l}3^{\mathrm{c}}, 55,71 \\
3^{\mathrm{A}}\end{array}$ & $\begin{array}{l}1 \\
1\end{array}$ & $\begin{array}{l}1.2 \\
1.2\end{array}$ \\
\hline Total groupe II & 2 & $\overline{2.4}$ \\
\hline
\end{tabular}


TABLEAU 5 (suite)

\begin{tabular}{|c|c|c|}
\hline Groupe & Numéro des échantillons & Pourcentage \\
\hline \multicolumn{3}{|l|}{ Groupe III : } \\
\hline $\begin{array}{l}7 \\
6,7,42^{\mathrm{E}} \\
54 \\
42^{\mathrm{E}}, 75 \\
6,42^{\mathrm{E}}, 54,75 \\
6,7,42^{\mathrm{E}}, 54,75\end{array}$ & $\begin{array}{r}17 \\
7 \\
2 \\
2 \\
1 \\
1\end{array}$ & $\begin{array}{r}20.4 \\
8.4 \\
2.4 \\
2.4 \\
1.2 \\
1.2\end{array}$ \\
\hline Total groupe III & 30 & 36.0 \\
\hline Groupe IV & 1 & 1.2 \\
\hline \multicolumn{3}{|l|}{ Groupe mixte : } \\
\hline $\begin{array}{l}6,7,42^{\mathrm{E}}, 47,64,75,82 \\
83^{\mathrm{A}}, 7 \\
42^{\mathrm{E}}, 83^{\mathrm{A}} \\
7,75,83^{\mathrm{A}}, 81 \\
3^{\mathrm{A}}, 6,7,42^{\mathrm{E}}, 47,53 \\
79,6,7,42^{\mathrm{E}} \\
52^{\mathrm{A}}, 3^{\mathrm{E}} \\
3^{\mathrm{A}}, 3^{\mathrm{C}}, 6,7,42^{\mathrm{E}}\end{array}$ & $\begin{array}{l}1 \\
2 \\
1 \\
1 \\
1 \\
1 \\
1 \\
2\end{array}$ & $\begin{array}{r}1.2 \\
2.4 \\
1.2 \\
1.2 \\
1.2 \\
1.2 \\
2.4 \\
12.0\end{array}$ \\
\hline & 10 & $\overline{12.0}$ \\
\hline Non typable & 34 & 40.8 \\
\hline Total échantillons & 81 & \\
\hline
\end{tabular}

\section{S u m m a r y}

212 samples of Iranian fresh, unsalted, white cheese produced in Tehran area have been studied for total count with special reference to coagulase positive staphylococci. 


\section{Bibliographie}

[1] Ghazvinian (R.), Maleki (M.) and Kohnéshahri (M.) (1972). - Nasal, Hand and throat carriage of coagulase-positive staphylococci in Food Handlers in Tehran. Pahlavi. Medical Journal, vol. 3, 38-45.

[2] Ghazvinian (R.), Farkhondeh (A.), Maleki (M.) et Kohnéshahri (M.) (1972). Dénombrement des germes totaux et des bactéries califormes des laits crus délivrés à la Centrale Laitière de Téhéran, et détermination du taux de contamination de ces laits en staphylocoques, coagulase-positive. Le Lait, $\mathrm{n}^{\circ}$ 511-512, janvier-février.

[3] Kohnéshahri (M.), Maleki (M.), Farkhondeh (A.) et Ghazvinian (R.) (1972). Dénombrement des germes totaux et des bactéries califormes des crèmes consommées dans la région de Téhéran et détermination du taux de contamination de ces crèmes en staphylocoques coagulase-positive. Le Lait, $\mathrm{n}^{\circ}$ 515-516, mai-juin.

[4] FARKHONDEH (A.) (1971). - Fromagerie artisanale en Iran. Tech. Lait., 714, 10 , septembre.

[5] Farkhondeh (A.) et SATtari (M.) (1971). - Conditions de la production de la collecte des traitements de la transformation, etc. des laits de brebis et de chèvre en Iran. Séminaire FIL, 27-30 avril, Madrid.

[6] FIL (1958). - Numération des micro-organismes des laits liquides et du lait en poudre. Norme FIL (3).

[7] Baird Parker (A. C.) (1962). - J. Appl. Bact., 25, 12.

[8] Thieulin (G.), Basille (D.), Pantaléon (J.), Rosset (R.), Gandon (Y.) et Petit (A.) (1966). - Recherche des staphylocoques pathogènes dans le lait et les produits laitiers. Le Lait, $\mathrm{n}^{\circ} 453-454,131$. 